\title{
Intraocular pressure after peribulbar anaesthesia: is the Honan balloon necessary?
}

\author{
J E Morgan, A Chandna
}

\begin{abstract}
Peribulbar anaesthetic blocks were administered to 70 patients and the intraocular pressure (IOP) measured immediately before and within 1 minute of the injections. In 10 patients IOPs were recorded at 1 minute intervals for 15 minutes after injection and then compared with the IOPs recorded in 60 patients after 5 or 10 minutes of Honan balloon ocular compression. The IOP rose significantly after injection in all patient groups and in some cases this increase was marked (over $50 \mathrm{~mm} \mathrm{Hg}$ in 10 patients). The IOP showed an equivalent drop after 5 or 10 minutes of ocular compression when compared with eyes that did not receive ocular compression. The Honan balloon does not appear to be necessary to reduce IOP in the 10 minutes following peribulbar injection. Furthermore, the occurrence of IOP peaks after peribulbar anaesthesia suggests that the balloon should be used with caution in eyes in which the ocular circulation may be compromised.
\end{abstract}

(Br f Ophthalmol 1995; 79: 46-49)

Peribulbar anaesthesia for intraocular surgery has gained in popularity over the last few years. It is relatively safe, ${ }^{1}$ easy to perform, and provides effective ocular akinesia and anaesthesia. ${ }^{2}$ Unfortunately, it tends to produce conjunctival chemosis and an increase in intraocular pressure (IOP) that may complicate ocular surgery. ${ }^{3}$ The Honan balloon ${ }^{4}$ is a popular method for the reduction of IOP and its use in retrobulbar anaesthesia has been widely reported. ${ }^{5-8}$ However, comparatively little is known of the pressure changes that occur after peribulbar anaesthesia and this has resulted in a variety of times being quoted as optimal for the application of the Honan balloon. In this study we have measured in detail the IOP changes after injection and used them as a baseline to see how IOP varies as a function of Honan balloon ocular compression.

\section{Methods}

We studied the IOP changes after peribulbar anaesthesia in 70 patients (one eye per patient) with a Tonopen XL (Biorad) following delivery of local anaesthetic for routine cataract surgery. Patients verbally consented to the procedure and the study conformed with the ethical standards of the United Bristol Health Care Trust ethics review committee.
Exclusion criteria included a history of glaucoma or ocular hypertension at the time of preoperative assessment or an axial length outside the range 19 to $25 \mathrm{~mm}$. All patients had their IOP measured immediately before the peribulbar anaesthetic. The patients were processed in two groups. Ten consecutive patients did not receive Honan balloon compression and for these the IOPs were measured at 1 minute intervals for 15 minutes after injection. The remaining 60 patients were randomised to receive external compression from the Honan balloon for either 5 or 10 minutes after injection, with IOP measurements made within the minute after injection and subsequently, within 1 minute of removal of the balloon. All patients then underwent routine extracapsular cataract extraction with lens implant.

The authors each used different anaesthetic solutions; $8 \mathrm{ml}$ of either $2 \%$ lignocaine (JM) or a $50 / 50$ mixture of lignocaine $2 \%$ and bupivacaine $0.5 \% \quad(\mathrm{AC})$ but the volume of delivered anaesthetic remained the same in all cases. All anaesthetic solutions were delivered percutaneously using a 27 gauge 1.5 inch retrobulbar needle, inferiorly at the junction of the medial two thirds and lateral one third of the inferior orbital rim and superiorly at the supratrochlear notch. Anaesthetic was delivered as a bolus of $3.5 \mathrm{ml}, 2 \mathrm{~cm}$ from the skin surface, and then as $0.5 \mathrm{ml}$ slowly injected as the needle was withdrawn, raising a small bleb in the subcutaneous tissues. Injections took approximately 90 seconds in total and post-injection IOP measurements were taken immediately after the last (superior) injection. The Honan balloon was then applied over a gauze swab placed on the eyelid, fastened with a head band and inflated to a pressure of $30 \mathrm{~mm} \mathrm{Hg}$. The three patient groups were compared by analysis of variance for age and pre-injection IOPs. Pre and post-injection IOPs were compared using the Wilcoxon rank sum test while other comparisons were made using paired $t$ test. All values are given as the mean (SD).

\section{Results}

The pressure changes after injection were studied in 70 patients. The mean age for all patients was 78.08 (SD 6.95) years of which 44 were female and 26 male. No significant difference in age or sex distribution was found between the different patient groups. All patients reported adequate anaesthesia throughout their operations. Ocular akinesia was satisfactory and operative difficulties were notable for one case of vitreous loss and 


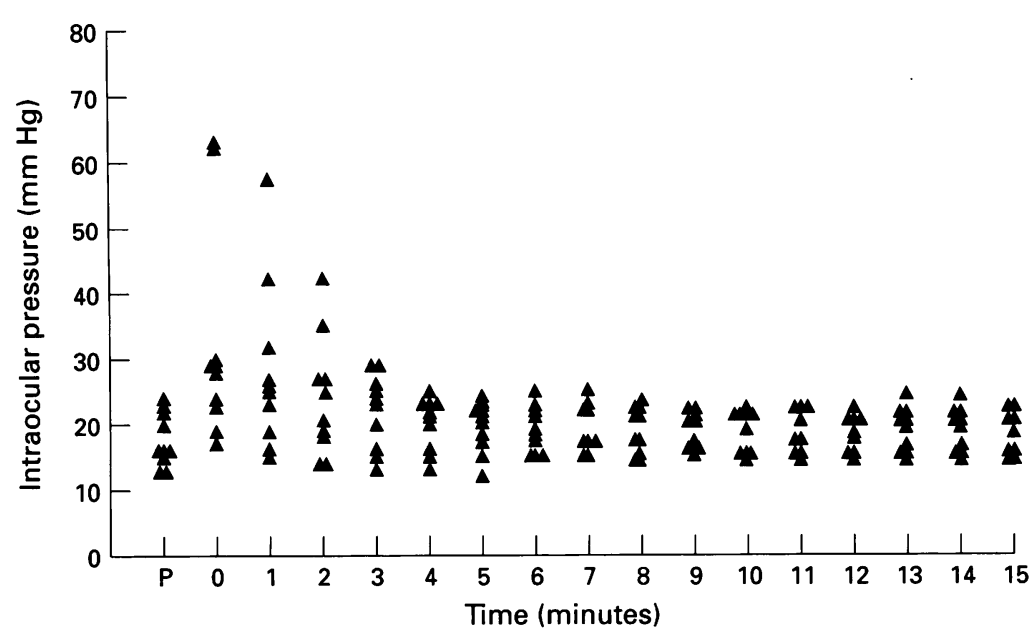

Figure 1 Scatter plot showing the intraocular pressures (IOPs) for the 15 minute period following injection for those patients that did not receive Honan balloon pressure.

$P$ indicates the IOPs recorded before peribulbar injection $(n=10)$. Time 0 represents the IOP measurement taken immediately after removal of the balloon; subsequent times are measured from this point $(n=10)$.

another that lasted 70 minutes. The investigators used different anaesthetic solutions but this did not produce any statistically different results between the various patient groups irrespective of balloon application times; the results from these groups are therefore treated together.

PRESSURE CHANGES IN THE 15 MINUTES AFTER PERIBULBAR INJECTION

Figure 1 shows the changes in intraocular pressure in the 15 minutes immediately after injection for the 10 patients who did not receive ocular compression from the Honan balloon. The corresponding values are summarised in Table 1. The IOP pressure increased significantly $(p=0.005)$ following injection (mean post-injection pressure 32.4 $(16.4) \mathrm{mm} \mathrm{Hg}$ ) with a minority of patients sustaining dramatic increases in IOP following injection (over $50 \mathrm{~mm} \mathrm{Hg}$ in three patients). However, the fall off in IOP occurring in the first 5 minutes after injection is rapid and by 4 minutes post-injection, IOPs no longer differed significantly from their pre-injection levels $(p=0.053)$. After this time, there was little change in IOP for any given patient. The low variability in IOP readings after 5 minutes emphasises the reliability of the Tonopen and suggests that its repeated use does not lower IOP.

PRESSURE CHANGES AFTER APPLICATION OF THE HONAN BALLOON

Figure 2 summarises the post-injection IOPs for the 30 patients who received Honan balloon pressure for 5 minutes after injection.

Table 1 The pre and post-injection intraocular pressures with standard deviations (SD) for the three groups under study

\begin{tabular}{llll}
\hline & Pre-injection & Post-injection & Post-balloon \\
\hline No balloon & $17 \cdot 80(4 \cdot 10)$ & $32 \cdot 40(16 \cdot 45)$ & $18 \cdot 00(3 \cdot 23)^{\star}$ \\
5 Minute balloon & $17 \cdot 40(3 \cdot 29)$ & $32 \cdot 13(11 \cdot 71)$ & $18 \cdot 07(5 \cdot 38)$ \\
10 Minute balloon & $17 \cdot 20(2 \cdot 99)$ & $38 \cdot 90(16 \cdot 00)$ & $15 \cdot 63(4 \cdot 69)$ \\
\hline
\end{tabular}

*Values correspond with IOP measurement at 15 minutes.

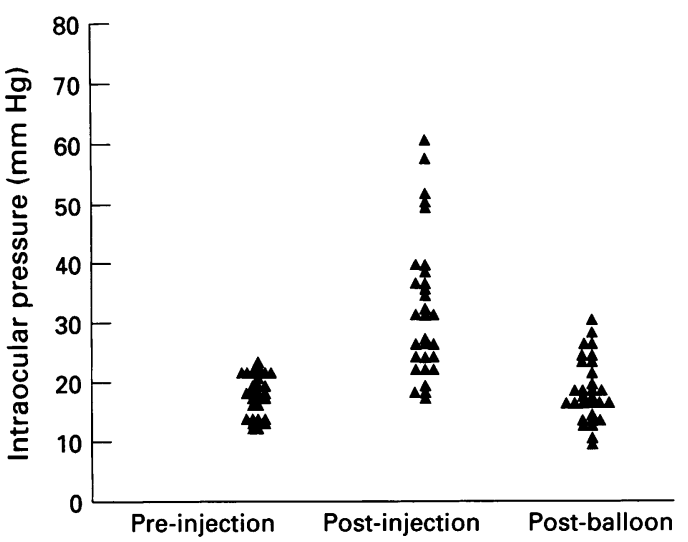

Figure 2 Scatter plot showing the distribution of intraocular pressures (IOPs) immediately before and just after peribulbar injection (pre-injection and post-injection, respectively) for those patients receiving Honan balloon ocular compression for 5 minutes. The points in the post Honan balloon group show the IOPs following release of the balloon after 5 minutes of application $(n=30)$.

Peribulbar injection produced a significant increase in IOP $(p<0.001)$ with a mean postinjection pressure of $32 \cdot 13(11 \cdot 71) \mathrm{mm} \mathrm{Hg}$ for which three patients had IOPs greater than $50 \mathrm{~mm} \mathrm{Hg}$. Application of the Honan balloon for 5 minutes after injection resulted in IOPs that did not differ significantly from the preinjection range $(p=0 \cdot 22)$. Figure 3 summarises the changes that occurred following peribulbar injection for the 30 patients who received 10 minutes of Honan balloon compression. Following injection there was a significant rise $(\mathrm{p}<0.001)$ in IOP (mean post-injection pressure $38.9(16.0) \mathrm{mm} \mathrm{Hg}$ ) with six patients having IOPs greater than $50 \mathrm{~mm} \mathrm{Hg}$. Analysis of variance did not reveal any difference between this rise and that in the 5 minute and no balloon groups $(p=0 \cdot 16)$. After 10 minutes of compression, IOPs did not differ significantly from the pre-injection level. One patient in this group sustained vitreous loss during subsequent surgery.

\section{Discussion}

As with other studies, ${ }^{12}$ 9-11 we found that a peribulbar approach provides effective ocular anaesthesia resulting in most cases, in an immobile eye that allows safe intraocular surgery. However, in all patient groups a

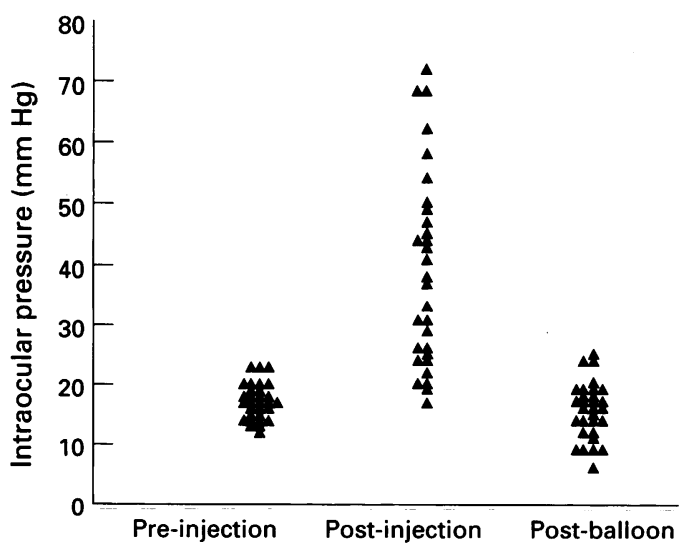

Figure 3 Scatter plot of the intraocular pressures for those patients receiving Honan balloon ocular compression for 10 minutes following peribulbar injection. Conventions as for Figure $2(n=30)$. 
significant minority of patients sustained a dramatic rise in IOP after injection, with 22 of 70 having IOPs at 1 minute post-injection of $40 \mathrm{~mm} \mathrm{Hg}$ and only 25 of 70 patients sustaining an IOP rise less than $10 \mathrm{~mm} \mathrm{Hg}$. Some of the pressure increases may have been higher than recorded given the tendency for the Tonopen to underestimate pressures greater than $30 \mathrm{~mm} \mathrm{Hg}{ }^{12} 13$

Only one other study ${ }^{3}$ has, to our knowledge, investigated the changes in IOP that follow peribulbar anaesthesia, showing in a sample of 36 patients that 27 had an average increase in IOP of $3.8 \mathrm{~mm} \mathrm{Hg}$ when measured 10 minutes after injection. Unfortunately, these results are not directly comparable with our work since the volume of injected anaesthetic varied from 7 to $12 \mathrm{ml}$ and the IOPs were not recorded immediately after injection. When immediate post-injection measurements have been made, as in a study of retrobulbar injections, ${ }^{6}$ a mean IOP increase of $4.4 \mathrm{~mm} \mathrm{Hg}$ has been reported. However, the injection volumes $(3-5 \mathrm{ml})$ used in this study were smaller than those routinely used in peribulbar injections and, at least in this volume range, the increase in IOP did not correlate with the volume of anaesthetic injected. The orbital volume has been estimated at $29.7 \mathrm{ml}^{14}$ so the increase in IOP we have observed with injected volumes of $8 \mathrm{ml}$ might have been expected.

Another important finding here is that without the Honan balloon the IOP decreased to the pre-injection pressure by 4 minutes and that the IOPs at 5 and 10 minutes in the no balloon group did not differ from those patients that had, respectively, 5 or 10 minutes of ocular compression. This contrasts with the findings of Ropo et al ${ }^{3}$ where the IOP was still $10 \%$ higher than the pre-injection pressure at 10 minutes in eyes that did not receive ocular compression. A possible explanation for this more prolonged rise in the IOP is that these workers used slightly larger injection volumes of $10 \mathrm{ml}$ (range $7-12 \mathrm{ml}$ ). Even so, Jay et alt measured the IOPs in patients following retrobulbar injections of only $4 \mathrm{ml}$ of anaesthetic and found an immediate post-injection rise of $6.1 \mathrm{~mm} \mathrm{Hg}$ that changed little after 7 to 10 minutes. Our study suggests that, following peribulbar delivery, the increased orbital pressure can be rapidly dissipated without external pressure. We were also unable to demonstrate the drop in IOP to less than the starting pressure that has been reported by other workers for ocular compression times of up to 10 minutes. Measurements following 10 minutes of ocular compression (using the Autopressor device) after peribulbar injection ${ }^{3}$ have shown decreases of $3.1 \mathrm{~mm} \mathrm{Hg}$ from the pre-injection pressure similar to those following retrobulbar anaesthesia ${ }^{7}$; larger reductions of up to 7-8 $\mathrm{mm} \mathrm{Hg}$ have been measured in cadaver eyes after Honan balloon application. ${ }^{8}$

Consideration of the effect of the Honan balloon in lowering IOP is also complicated by the volume and site of the injected anaesthetic. When no injections are made, the mean drop in IOP following a 30 minute balloon application is $5.2 \mathrm{~mm} \mathrm{Hg}^{5}$ and $6.97 \mathrm{~mm} \mathrm{Hg}$ after
40 minutes. ${ }^{15}$ The absence of such a decrease from the pre-injection pressure in our study might be expected considering the increase in IOP immediately after injection. We must stress that our study does not address the marked reductions in IOP (to $5 \mathrm{~mm} \mathrm{Hg}$ or less) that have been demonstrated with balloon application times greater than 40 minutes following retrobulbar blocks. ${ }^{16}$

Analysis of variance did not reveal any difference between the pre-injection IOPs $(p=0 \cdot 88)$. However, an unexpected finding was that eight of the 70 patients had pre-injection IOPs just greater than the upper limit of normal (mean 22.7 (0.49) $\mathrm{mm} \mathrm{Hg}$ ). All of these patients had IOPs within the normal range at preoperative assessment and a negative history of ocular hypertension. One possible explanation is that an increase in the tone of orbicularis increased during IOP measurements; this was a significant factor in one patient whose IOP dropped from $28 \mathrm{~mm} \mathrm{Hg}$ to $25 \mathrm{~mm} \mathrm{Hg}$ after injection, secondary to the relaxation of orbicularis. Alternatively, it is possible that pupillary dilatation may have compromised aqueous outflow. It is difficult in this study to estimate the importance of this factor since gonioscopy was not performed following dilatation. Since these IOPs were only slightly outside the normal range, we have not excluded them from this study. It is important to note that the postinjection IOP rise sustained by patients with a high starting IOP (mean 13.86 (14.79) $\mathrm{mm} \mathrm{Hg})$ did not differ significantly $(\mathrm{p}=0.45)$ from that seen in patients with a normal starting IOP (mean 18.32 (14.59) $\mathrm{mm} \mathrm{Hg}$ ).

Measurement of the IOP change following application of the Honan balloon in cadaver eyes has shown that IOP can be significantly higher than the $30 \mathrm{~mm} \mathrm{Hg}$ recorded by the Honan balloon. ${ }^{8}$ The IOP reaches a peak within a few seconds of balloon application and, although it declines over the next 5 minutes of application, can still remain at a considerable level (>60 mm Hg). Pressure peaks of up to $130 \mathrm{~mm} \mathrm{Hg}$ have been recorded in eyes with starting IOPs greater than $30 \mathrm{~mm} \mathrm{Hg}$ highlighting the problems that could arise in eyes in which the ophthalmic circulation is compromised. ${ }^{8}$ In rabbit eyes, for example, application of the Honan balloon results in a significant decrease in ocular and optic nerve head blood flow. ${ }^{17}$ The lack of recorded cases of arterial occlusion following its use in humans might, therefore, seem surprising ${ }^{16}$ were it not for evidence that the ocular circulation may compensate for these imposed hardships. In the experimental model, ocular blood flow increases following the removal of externally applied pressure ${ }^{17} 18$ and in humans, measurement of the systolic retinal and ciliary body perfusion pressure by oculodynamography ${ }^{5}$ has shown that this can increase following 25 minutes of ocular compression (at 30-50 $\mathrm{mm} \mathrm{Hg}$ ). Measures can also be taken by the anaesthetist to safeguard against an excessive post-injection pressure rise. Zabel et al ${ }^{19}$ have shown that for the retrobulbar method, when the balloon is 
applied before anaesthesia, a greater postoperative pressure drop is seen when compared with pressure application after retrobulbar injection ( $4.7 \mathrm{~mm} \mathrm{Hg} v 2.24 \mathrm{~mm} \mathrm{Hg}$ ). A similar protective effect is also seen with intravitreal injections. ${ }^{20}$ An additional, but unproved method to limit the pressure rise is to fractionate the injection volumes around the globe. ${ }^{3}$

This study describes the increases in IOP occurring following peribulbar anaesthesia that have not been reported previously and questions the role of the Honan balloon in producing a rapid decrease in IOP immediately following peribulbar injection. We have not addressed the propensity of the balloon to decrease post-injection conjunctival chemosis and lid swelling; subjectively this can be a beneficial effect of balloon application in many patients. Our results have demonstrated the occurrence of very high IOPs in a significant proportion of patients undergoing peribulbar anaesthesia which have important implications when considering surgery for patients whose ocular circulation may be compromised. In these cases, one might consider pharmacological lowering of the IOP before surgery, ${ }^{21}$ fractionation of the anaesthetic injection, ocular compression before injection, or even avoidance of Honan balloon compression altogether. Our results are based on the widely used injection volume of $8 \mathrm{ml}$. One would predict, by comparison with the smaller injection volumes used in retrobulbar anaesthesia, that a smaller post-injection pressure rise might be achieved with a smaller volume of injected anaesthetic.

1 Davis DB, Mandel MR. Posterior peribulbar anesthesia: an alternative to retrobulbar anesthesia. $\mathcal{F}$ Cataract Ref Surg 1986; 12: $182-4$
2 Murdoch IE. Peribulbar versus retrobulbar anaesthesia. Eye 1990; 4: 445-9.

3 Ropo A, Ruusuvaara P, Paloheimo M, Maunuksela EL, Nikki P. Effect of ocular compression (Autopressor) on intraocular pressure in periocular anaesthesia. Acta Ophthalmol Copenh 1990; 68: 227-9.

4 Honan PR. Some observations on the use of a pneumatic eye softener for intraocular surgery. Contact Intraoc Lens Med F 1979; 5: 78-80.

5 Davidson B, Kratz RP, Mazzocco TR, Maloney WF. An evaluation of the Honan intraocular pressure reducer. $\mathrm{Am}$ Intraocular Implant Soc f 1979; 5: 237.

6 Jay WM, Carter H, Williams B, Green K. Effect of applying the Honan intraocular pressure reducer before cataract surgery. Am $\mathcal{F}$ Ophthalmol 1985; 100: 523-7.

7 Quist LH, Scott Stapleton S, Mcpherson SD. Preoperative use of the Honan intraocular lens intraducer. $A m \mathcal{F}$ Ophthalmol 1983; 95: 536-8.

8 McDonnell PJ, Quigley HA; Maumenee AE, Stark WJ, Hutchins GM. The Honan intraocular pressure reducer. Arch Ophthalmol 1985; 103: 422-5.

9 Wang HS. Peribulbar anesthesia for ophthalmic procedures. f Cataract Ref Surg 1988; 14: 441-3.

10 Bloomber LB. Administration of periocular anesthesia. f Cataract Ref Surg 1986; 12: 677-9.

11 Weiss JL, Deichman CB. A comparison of retrobulbar and periocular anesthesia for cataract surgery. Arch Ophthalmol 1989; 107: 96-8.

12 Kao SF, Lichter PR, Bergstrom TJ, Rowe S, Musch DC. Clinical comparison of the Oculabe Tono-pen to the Goldmann applanation tonometer. Ophthalmology 1987; 94: 1541-4.

13 Frenkel REP, Hong YJ, Shin DH. Comparison of the Tono-pen to the Goldmann applanation tonometer. Arch Ophthalmol 1988; 106: 750-3.

14 Whitnall SE. One a tubercle on the malar bone and on the lateral attachments of the tarsal plates. F Anat Physiol 1911; 45: 426.

15 Constable PH, Porter EJB. Extraocular compression prior to cataract surgery: time course of reduction and subsequent recovery of intraocular pressure. Eye 1993; 7: 731-4.

16 Martin NF, Stark WJ, Maumenee AE, Bruner WE, Rosenblum $P$. Use of the Honan intraocular pressure reducer at the Wilmer institute. Ophthalmic Surg 1982; 13: $101-3$.

17 Jay WM, Aziz MZ, Green K. Effect of honan intraocula pressure reducer on ocular and optic nerve blood flow in phakic rabbit eyes. Acta Ophthalmol Copenh 1986; 64: 52-7.

18 Jay WM, Aziz MZ, Green K. Effect of digital massage on intraocular pressure and ocular and optic nerve blood intraocular pressure and octa Ophthalmol Copenh 1986; 64: 58-62.

19 Zabel RW, Clarke WN, Shirley SY, Rock W. Intraocular pressure reduction prior to retrobulbar injection of anaesthetic. Ophthalmic Surg 1988; 19: 868-71.

20 Morlet N, Young SH. Prevention of intraocular pressure rise following intravitreal injection. Br f Ophthalmol 1993; 77: 572-3.

21 Mirakur R, Shepherd W. Intraocular pressure changes with propofol (Diprivan): comparison with thiopentone. Postgrad Med 1985; 61 (suppl 3): 41-4. 